\title{
Utilization of sialic acid as a coreceptor is required for reovirus-induced biliary disease
}

\author{
Erik S. Barton, ${ }^{1,2}$ Bryan E. Youree, ${ }^{2,3}$ Daniel H. Ebert, ${ }^{1,2}$ J. Craig Forrest, ${ }^{1,2}$ \\ Jodi L. Connolly, ${ }^{1,2}$ Tibor Valyi-Nagy, ${ }^{2,4}$ Kay Washington, ${ }^{4}$ J. Denise Wetzel, 2,5 \\ and Terence S. Dermody ${ }^{1,2,5}$ \\ ${ }^{1}$ Department of Microbiology and Immunology, \\ ${ }^{2}$ Lamb Center for Pediatric Research, \\ ${ }^{3}$ Department of Medicine, \\ ${ }^{4}$ Department of Pathology, and \\ ${ }^{5}$ Department of Pediatrics, Vanderbilt University School of Medicine, Nashville, Tennessee, USA
}

Infection of neonatal mice with some reovirus strains produces a disease similar to infantile biliary atresia, but previous attempts to correlate reovirus infection with this disease have yielded conflicting results. We used isogenic reovirus strains T3SA- and T3SA+, which differ solely in the capacity to bind sialic acid as a coreceptor, to define the role of sialic acid in reovirus encephalitis and biliary tract infection in mice. Growth in the intestine was equivalent for both strains following peroral inoculation. However, T3SA+ spread more rapidly from the intestine to distant sites and replicated to higher titers in spleen, liver, and brain. Strikingly, mice infected with T3SA+ but not T3SA- developed steatorrhea and bilirubinemia. Liver tissue from mice infected with $\mathrm{T} 3 \mathrm{SA}+$ demonstrated intense inflammation focused at intrahepatic bile ducts, pathology analogous to that found in biliary atresia in humans, and high levels of T3SA+ antigen in bile duct epithelial cells. T3SA+ bound 100-fold more efficiently than T3SA- to human cholangiocarcinoma cells. These observations suggest that the carbohydrate-binding specificity of a virus can dramatically alter disease in the host and highlight the need for epidemiologic studies focusing on infection by sialic acid-binding reovirus strains as a possible contributor to the pathogenesis of neonatal biliary atresia.

J. Clin. Invest. 111:1823-1833 (2003). doi:10.1172/JCI200316303.

\section{Introduction}

Reoviruses infect most mammalian species, including humans, but reovirus-induced disease is limited to the very young (1). Reovirus infections of newborn mice provide a powerful experimental system for studying the role of virus-receptor interactions in the pathogenesis of viral disease. Following replication in intestinal lymphoid cells, all three reovirus serotypes spread through the blood to distant sites including heart, brain, liver,

Received for publication June 27, 2002, and accepted in revised form April 9, 2003.

Address correspondence to: Terence S. Dermody, Lamb Center for Pediatric Research, D7235 MCN, Vanderbilt University School of Medicine, Nashville, Tennessee 37232, USA.

Phone: (615) 343-9943; Fax: (615) 343-9723;

E-mail: terry.dermody@vanderbilt.edu.

Erik S. Barton's present address is: Department of Pathology, Washington University School of Medicine, St. Louis, Missouri, USA. Jodi L. Connolly's present address is: Heller, Ehrman, White, and McAuliffe LLP, San Diego, California, USA.

Tibor Valyi-Nagy's present address is: Department of Pathology, University of Illinois, Chicago, Illinois, USA.

Erik S. Barton and Bryan E. Youree contributed equally to this work.

Conflict of interest: The authors have declared that no conflict of interest exists.

Nonstandard abbreviations used: serotype 1 (T1); oily hair effect (OHE); junctional adhesion molecule-1 (JAM1); murine erythroleukemia cells (MEL cells); murine L929 cells (L cells). spleen, and kidneys (2). However, reovirus serotypes differ in the pathogenesis of CNS infections (2). Serotype 1 (T1) strains enter the CNS via hematogenous routes, replicate within ependymal cells, and cause self-limiting hydrocephalus (3). In sharp contrast, serotype 3 (T3) strains enter the CNS by neural routes, spread from the myenteric plexus along the vagus nerve, and ultimately reach the vagus nucleus in the brain stem (4). Once in the CNS, T3 strains replicate in defined populations of neurons (5) and cause lethal encephalitis that is associated with neuronal apoptosis (6).

In addition to encephalitis, infection with a subset of $\mathrm{T} 3$ reovirus strains is associated with development of oily hair effect $(\mathrm{OHE})(7,8)$, a syndrome associated with viral replication in intrahepatic bile duct epithelium, biliary obstruction, and fat malabsorption $(7,9-11)$. OHE resembles human biliary atresia, an acute and progressive inflammatory destruction of bile duct epithelium that is the leading cause of liver transplantation in children (12-15). Numerous studies have attempted to correlate reovirus seropositivity with biliary atresia, with disparate results (16-23). However, reovirus RNA was detected in $50-78 \%$ of one group of patients with obstructive liver disease, while only $12 \%$ of control samples contained reovirus RNA (24). This correlation was statistically significant, but not absolute, suggesting 
that either only a subset of reovirus strains are capable of causing biliary atresia or additional host factors contribute to disease penetrance.

Experiments using genetic reassortant viruses indicate that the route of spread $(25,26)$, CNS tropism $(27,28)$, and biliary disease (7) segregate with the $S 1$ gene segment, which encodes the viral attachment protein known as $\sigma 1(29,30)$. Most T3 $\sigma 1$ proteins bind terminal sialic acid on glycosylated cellular proteins (31-35) using sequences in the fibrous $\sigma 1$ tail domain (36-38). However, some naturally occurring $\mathrm{T} 3$ strains do not bind sialic acid and are presumed to be incapable of binding any carbohydrate (39). T1 $\sigma 1$ binds a non-sialic acid carbohydrate that has not been identified (40), also using sequences in the tail domain (38). In addition to the carbohydrate-binding domains in the o1 tail, the globular head domains of both $\mathrm{T} 1$ and $\mathrm{T} 3 \mathrm{\sigma} 1 \mathrm{use}$ junctional adhesion molecule-1 (JAM1) as a cell-surface receptor (41). JAM1 is a member of the immunoglobulin superfamily and influences both tight junction formation (42) and leukocyte transmigration across endothelium $(43,44)$.

The finding that JAM1 serves as a serotype-independent reovirus receptor suggests that o1-JAM1 interactions are not solely responsible for defining reovirus tropism in vivo, thereby pointing to a potential role for carbohydrate coreceptors in this process. Since almost all detailed studies of $\mathrm{T} 3$ reovirus pathogenesis have used sialic acid-binding strains $(4,5,25,26,28)$, little is known about the role of sialic acid in determining reovirus tropism and disease in the infected host. Some cultured cell lines, including murine erythroleukemia cells (MEL cells), support binding and infection of only those reovirus strains that bind sialic acid $(36,37)$. For other cell types, such as HeLa cells, the capacity to bind sialic acid significantly enhances the efficiency of viral adsorption and infection (45). However, murine L929 cells (L cells) do not require sialic acid binding for efficient infection $(32,45)$. It is possible that distinct cell types exist in vivo that mimic these differential requirements for sialic acid binding.

To define the contribution of sialic acid binding to the pathogenesis of $\mathrm{T} 3$ reovirus disease, we infected newborn mice with reovirus strains T3SA- and T3SA+ and compared mortality, viral replication, tropism, pathologic injury, and disease outcome. The $\sigma 1$ protein of T3SA- does not bind sialic acid, whereas the $\sigma 1$ protein of T3SA+ possesses a single amino acid substitution that confers high-avidity binding to this carbohydrate (45). We found that in comparison to T3SA-, strain $\mathrm{T} 3 \mathrm{SA}+$ spreads more rapidly from the intestine to distal sites of infection, including the CNS. In addition, T3SA+, but not T3SA-, infects bile duct epithelial cells and causes obstructive liver disease in mice. These results indicate that the capacity of reovirus to bind sialic acid alters the reovirus-host interaction, resulting in distinct disease phenotypes. Furthermore, these findings suggest that infection of human neonates with the subset of reovirus strains capable of binding sialic acid may lead to biliary atresia.

\section{Methods}

Cells, viruses, and antibodies. L cells and MEL cells were grown in either suspension or monolayer cultures as previously described (37). Mz-Cha-1 human cholangiocarcinoma cells derived from a gallbladder adenocarcinoma metastasis were cultured in CMRL 1066 medium (Invitrogen Corp., Carlsbad, California, USA) as described (46). Reovirus strains T3C44-SA- (T3SA-) and $\mathrm{T} 3 \mathrm{C} 44-\mathrm{MA}-\mathrm{SA}+(\mathrm{T} 3 \mathrm{SA}+)$ were generated by genetic reassortment of strains T3C44 and T3C44-MA, respectively, with strain type 1 Lang (45). T3SA- contains the $S 1$ gene of T3C44, which does not bind sialic acid $(37,39)$, whereas $\mathrm{T} 3 \mathrm{SA}+$ contains the $S 1$ gene from T3C44-MA, which binds sialic acid by virtue of a single substitution (Leu ${ }^{204} \rightarrow$ Pro) in the o1 tail (37). The remaining nine genes of both T3SA- and T3SA + are derived from strain type 1 Lang (45). Purified virion preparations were made using second- or third-passage L cell lysate stocks of reovirus that had been plaquepurified twice (47). Polyclonal rabbit anti-reovirus serum was obtained as previously described (48). Antireovirus serum was heat-inactivated by incubation at $56^{\circ} \mathrm{C}$ for 60 minutes.

Mice and inoculations. ND4 Swiss Webster mice (2-3 days old; Harlan, Indianapolis, Indiana, USA), at an average weight of $2 \mathrm{~g}$, were inoculated perorally or intracranially with purified virus diluted in gelatin saline. Prior to inoculation, mice from simultaneously delivered litters were pooled and randomized into litters of eight to eleven mice. For peroral inoculations, 50 $\mu \mathrm{l}$ of virus was delivered into the stomach by passage of a 0.61-mm polyethylene catheter (Becton, Dickinson and Co., Sparks, Maryland, USA) through the esophagus (49). For intracranial inoculations, $5 \mu$ l of virus was delivered into the right cerebral hemisphere using a Hamilton syringe and a 30-gauge needle (BD Biosciences, Palo Alto, California, USA) (50). At various times after inoculation, mice were euthanized and organs were harvested into $2 \mathrm{ml}$ of gelatin saline and homogenized by freezing, thawing, and sonication. Viral titer in organ homogenates was determined by plaque assay using $L$ cells (51). The small and large intestines were harvested in their entirety for determination of viral titer in the intestine.

The $\mathrm{LD}_{50}$ of reovirus was determined using litters of mice inoculated with a single dose of reovirus either perorally or intracranially and monitored daily for survival for 21 days after inoculation. Moribund mice were euthanized. $\mathrm{LD}_{50}$ values were calculated using the method of Reed and Muench (52). All animal experiments were performed using protocols consistent with Association for Assessment of Laboratory Animal Care regulations.

Quantitation of serum alkaline phosphatase and bilirubin levels. At various times after inoculation, mice were anesthetized and decapitated. Blood was collected and allowed to coagulate, and serum was separated by centrifugation. Sera were stored at $-20^{\circ} \mathrm{C}$, protected from light, and submitted in batches to the 
Oppenheimer United Reference Laboratory (Nashville, Tennessee, USA) for determination of total bilirubin and alkaline phosphatase levels.

Histology and immunobistochemical staining for reovirus antigen. At various times after inoculation, mice were euthanized and tissues were harvested. Organs were fixed in $10 \%$ buffered formalin at $4^{\circ} \mathrm{C}$ for $24-48$ hours, then embedded in paraffin and thin-sectioned using standard histologic techniques. Deparaffinized tissue was stained using hematoxylin and eosin prior to microscopic examination.

For immunohistochemical staining, deparaffinized tissue was rehydrated by incubation in PBS for 20 minutes at room temperature. Endogenous peroxidase was quenched by incubation for 30 minutes in $0.3 \%$ peroxide in methanol. Tissues were blocked by incubation for 20 minutes in PBS containing 1.5\% normal goat serum. Tissues were incubated for 30 minutes in protein A affinity-purified anti-reovirus serum (1:800 in blocking solution) that was first preadsorbed against methanol-fixed L cell monolayers. Antigen-positive cells were visualized using avidin/biotin-conjugated HRP and 3,3'-diaminobenzadine (DAB) substrate according to the manufacturer's instructions (Vector Laboratories Inc., Burlingame, California, USA). After $\mathrm{DAB}$ staining, tissues were rinsed in deionized water and counterstained for 45 seconds with hematoxylin and mounted under coverslips.

$R T$-PCR and restriction digestion. Viral RNA was purified from homogenized livers of infected mice or second-passage lysate stocks using TRI reagent (Molecular Research Center Inc., Cincinnati, Ohio, USA). RT-PCR was performed by mixing either $500 \mathrm{ng}$ of viral RNA or $5 \mu$ l of a 1:50 dilution of total organ RNA with 500 ng of each primary PCR primer diluted to 30 $\mu \mathrm{l}$ in water. RNA and primers were incubated at $94^{\circ} \mathrm{C}$ for 2 minutes and snap-cooled on ice. The primary PCR reaction volume was $50 \mu \mathrm{l}$, with final concentrations of $1 \times$ Taq buffer (Promega Corp., Madison, Wisconsin, USA), $1.5 \mathrm{mM} \mathrm{MgCl}_{2}, 200 \mu \mathrm{M}$ each dNTP, 15 $\mathrm{U}$ avian myeloblastosis virus reverse transcriptase (Promega Corp.), and 2.5 U Taq DNA polymerase (Promega Corp.). Reverse transcription was performed at $42^{\circ} \mathrm{C}$ for 1 hour, followed by incubation at $94^{\circ} \mathrm{C}$ for 1 minute. Samples were then amplified for 22 cycles of a protocol consisting of $94^{\circ} \mathrm{C}$ for 20 seconds, $53^{\circ} \mathrm{C}$ for 2 minutes, and $72^{\circ} \mathrm{C}$ for 5 minutes, followed by an additional incubation at $72^{\circ} \mathrm{C}$ for 20 minutes. Nested PCR was performed using $500 \mathrm{ng}$ of each secondary primer and $5 \mu \mathrm{l}$ of the primary PCR amplification product $(782 \mathrm{bp})$ in a final volume of $50 \mu \mathrm{l}$. Secondary amplification was performed using 20 cycles of an identical protocol. The oligonucleotide primers for the primary PCR were: forward, 5'-GATGCTCAACTTGCAATCTCC- $3^{\prime}$, and reverse, $5^{\prime}$-TGTACCCTCCAATTCAGCCC- $3^{\prime}$. The oligonucleotide primers for the secondary PCR were: forward, 5 '-CCACTGAGCTATCAACTCTGAC- 3 ', and reverse, 5'-TCCTATCCACATGCTCTGCC-3'. Restriction digestion was performed using BstNI (New England Biolabs Inc., Beverly, Massachusetts, USA). Digested secondary PCR products were resolved by agarose gel electrophoresis and visualized by ethidium bromide staining. Bst $\mathrm{NI}$ digestion results in cleavage of the T3SA+ S1 PCR secondary product after the cytosine at bp 190, yielding fragments of 190 and $355 \mathrm{bp}$, while the T3SA- S1 PCR product is not cleaved by this enzyme.

Assessment of virus growth in L cells and MEL cells. Homogenized organ samples $(200 \mu \mathrm{l})$ harvested at days 4,8 , and 12 after peroral inoculation were passaged in L cells $\left(6 \times 10^{6}\right)$ at $37^{\circ} \mathrm{C}$ for 72 hours, and lysates were obtained by three cycles of freezing and thawing. Tissue culture medium was aspirated from cells, and virus inoculum $(150 \mu \mathrm{l})$ was adsorbed to cells $\left(2 \times 10^{5}\right)$ at room temperature for 1 hour. The inoculum was removed, cells were washed with $1 \mathrm{ml}$ of PBS, and $1 \mathrm{ml}$ of complete medium was added. Cells were incubated at $37^{\circ} \mathrm{C}$ for various intervals, followed by three cycles of freezing and thawing to release progeny virions. Titers of infectious virus in lysates were determined by plaque assay using L cells (51).

Virus binding assays. L cells or Mz-Cha- 1 cells were released from culture flasks using PBS with 0.02\% EDTA and brisk pipetting. Detached cells were resuspended at $2 \times 10^{6}$ per $\mathrm{ml}$ in PBS and either mocktreated or incubated at $37^{\circ} \mathrm{C}$ for 1 hour with $1 \mathrm{U}$ per $\mathrm{ml}$ Clostridium perfringens neuraminidase (SigmaAldrich, St. Louis, Missouri, USA) to remove cell-surface sialic acid. Neuraminidase reactions were terminated by adding 10 volumes of ice-cold FACS wash buffer (PBS, $10 \%$ FBS, $1 \%$ BSA, and $0.02 \%$ sodium azide) and centrifuging at $500 \mathrm{~g}$ for five minutes. Cells were resuspended in FACS wash buffer and incubated at room temperature for 30 minutes with $0,10^{3}$, $10^{4}$, or $10^{5}$ particles per cell of either T3SA- or T3SA+. Binding reactions were performed using U-bottom microtiter plates with $5 \times 10^{5}$ cells in a volume of 0.1 $\mathrm{ml}$. Unbound virus was removed by two washes with $0.15 \mathrm{ml}$ cold FACS wash buffer and centrifugation for two minutes at $500 \mathrm{~g}$. Cells were incubated with primary antibody (1:1,000 dilution of preimmune or hyperimmune serum generated against reovirus strain type 1 Lang) and secondary antibody (1:500 dilution of goat anti-rabbit phycoerythrin-conjugated Fab fragments) at $4^{\circ} \mathrm{C}$ for 30 minutes, followed by washing with FACS wash buffer. Cells were fixed in $2 \%$ paraformaldehyde and analyzed using a FACSCalibur flow cytometer (BD Biosciences Immunocytometry Systems, San Jose, California, USA).

\section{Results}

Growth of T3SA- and T3SA+ in mice following peroral inoculation. We reasoned that sialic acid binding might alter any of several steps in reovirus pathogenesis, including (a) viral replication in the intestine, (b) spread from the intestine to secondary organs, or (c) the specificity of viral tropism for different cell types. To determine the effect of sialic acid binding on viral 


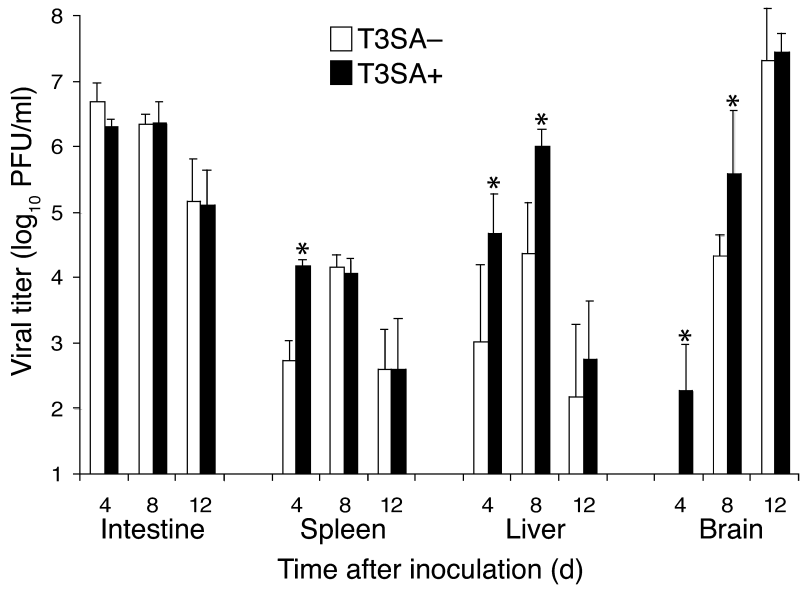

Figure 1

Growth ofT3SA- and T3SA+ in mice after peroral inoculation. ND4 Swiss Webster mice, 2-3 days old, were inoculated perorally with $2.5 \times 10^{3} \mathrm{PFU}$ of either T3SA- or T3SA+ in a volume of $50 \mu \mathrm{l}$. At the indicated times after inoculation, mice were euthanized and organs were collected. Organs were homogenized by sonication, and titers of virus present in homogenates were determined by plaque assay. Each bar represents the average viral titer from three mice. Error bars indicate SEM. ${ }^{*} P<0.05$ by Student $t$ test.

growth in the intestine and spread to secondary sites of replication, newborn ND4 Swiss Webster mice were inoculated perorally with $2.5 \times 10^{3} \mathrm{PFU}$ of T3SA- or $\mathrm{T} \mathrm{SA}+$. Organs were harvested at various times after infection, and infectious virus in each organ was quantitated (Figure 1). We found that both strains produced equivalent titers of virus in the intestine at 4,8 , and 12 days after inoculation. Two days following oral inoculation, the number of antigen-positive mononuclear cells in the duodenal lamina propria of mice infected with T3SA- or T3SA+ were similar (data not shown), consistent with the equivalent viral titer at this site at later times. Four days after inoculation, mice inoculated with T3SA- or T3SA+ displayed moderate inflammatory infiltrates in the duodenum that were qualitatively equivalent (data not shown). These data indicate that the efficiency and specificity of reovirus replication in the intestine is not altered by the capacity to bind sialic acid.

In contrast, we observed that viral titer in spleen, liver, and brain at day 4 was approximately 50 -fold higher in mice infected with $\mathrm{T} 3 \mathrm{SA}+$ than in mice infected with T3SA-. By day 8 , the titer of T3SA- in spleen had increased to the level of T3SA+, and titers of both strains were equivalently reduced by day 12 . In liver, more T3SA+ than T3SA- was detectable at days 4 and 8 , but titers were equivalent for the two strains by day 12. Although titers of T3SA- were not detectable in the brain on day 4, T3SA+ was readily detected at this timepoint and remained elevated relative to T3SA- at day 8. Unlike titers in the intestine and spleen, titers of virus in the brain were greater on day 12 than at earlier timepoints, indicating progressive CNS replication by both strains. Twelve days after inoculation with either strain, infected animals exhibited circling behavior, hind limb paralysis, and seizures, consistent with fulminant encephalitis (1). These results suggest that the capacity to bind sialic acid either enhances reovirus spread from the intestine to distal sites of replication or influences the growth of reovirus once these sites are reached.

Growth of T3SA- and T3SA+ in mice following intracranial inoculation. To determine whether increased titer of $\mathrm{T} 3 \mathrm{SA}+$ in the CNS following peroral inoculation is due to enhanced neuroinvasion or accelerated replication at that site, we assessed the capacity of strains T3SAand T3SA+ to replicate in the brain following intracranial inoculation (Figure 2). Newborn mice were inoculated intracranially with $10^{3} \mathrm{PFU}$ of each strain, and brain was harvested at $1,12,24,36$, or 48 hours after inoculation. At 1 hour after inoculation, viral titer in brain tissue of infected mice was approximately tenfold less than input virus. By 12 hours after inoculation, however, viral titers for both strains were approximately tenfold greater than input virus. By 48 hours after inoculation, viral titers for both strains were 100- to 1,000 -fold above input. These findings indicate that sialic acid binding does not dramatically alter the efficiency of acute CNS replication of $\mathrm{T} 3$ reovirus strains. These results also suggest that increased viral titers at early timepoints in brain tissue of mice perorally inoculated with T3SA+ (Figure 1) represent a sialic acid-mediated enhancement of T3SA+ spread from the intestine to the CNS.

Genotype and phenotype of T3SA - and T3SA+ remain stable in vivo. The observation that titers of T3SA- eventually reach a level equivalent to those of T3SA+ in the spleen, liver, and brain suggested that selection for sialic acid-binding variants might occur during infection in the host. To determine whether T3SA- and T3SA+

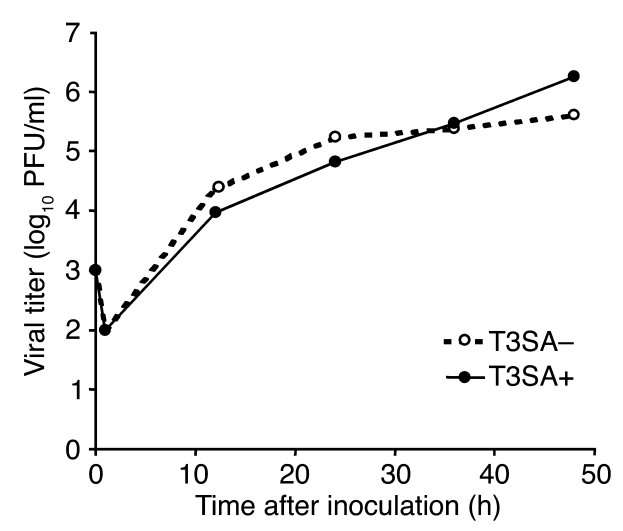

Figure 2

Growth of T3SA- and T3SA+ in mice after intracranial inoculation. ND4 Swiss Webster mice, 2-3 days old, were inoculated intracranially with $1 \times 10^{3} \mathrm{PFU}$ of either T3SA- or T3SA+ in a volume of $5 \mu \mathrm{l}$. At the indicated times after inoculation, mice were euthanized and brains were collected. Brain tissue was homogenized by sonication, and titers of virus present in homogenates were determined by plaque assay. Each data point represents the average viral titer from two to four brains. 


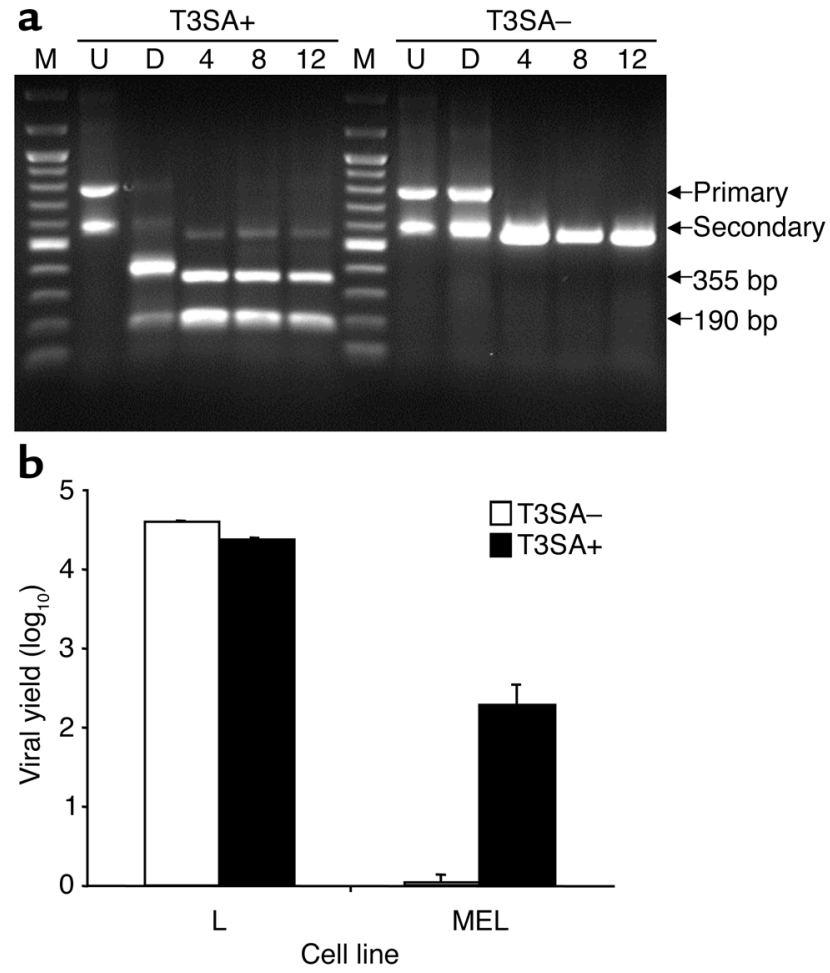

are genetically stable during multiple rounds of replication in vivo, we used a Bst NI restriction site polymorphism in the T3SA+ S1 gene that is responsible for the sialic acid-binding Leu ${ }^{204} \rightarrow$ Pro mutation in the o1 tail (Figure 3a). Total RNA was extracted from infected livers at 4,8 , and 12 days after inoculation. The viral $S 1$ gene was amplified from control virus stocks or liver RNA using nested RT-PCR and digested with $B s t \mathrm{NI}$, which cleaves the T3SA+ but not the T3SA-S1 fragment. We found that no BstNI-cleavable S1 fragment could be isolated from livers infected with T3SAat any timepoint, while all $\mathrm{S} 1$ amplicons from mice infected with T3SA+ remained BstNI-sensitive. These findings indicate that the sialic acid-binding mutation present in $\mathrm{T} 3 \mathrm{SA}+$ is stable for up to 12 days in vivo and that selection of this mutation does not occur during infection with T3SA- for the interval assessed.

To confirm that the sialic acid-binding phenotypes of these strains are maintained in vivo, organ lysates were assayed for the capacity to infect L cells and MEL cells (Figure 3b). Although both T3SA+ and T3SA- grow efficiently in $L$ cells (45), only sialic acid-binding reovirus strains are capable of infecting MEL cells (37). Consistent with the genetic stability of T3SA- and T3SA+, we found that virus in organ lysates of mice infected with T3SAreplicated efficiently in L cells but not in MEL cells, whereas virus in organ lysates of mice infected with $\mathrm{T} 3 \mathrm{SA}+$ replicated efficiently in both cell types. These data indicate that mutations altering the carbohydrate-binding specificity of T3SA- and T3SA+ do not occur during the course of replication within the host, indicating that pathogenic phenotypes differing between these two strains can be biochemically linked to sialic acid binding.

\section{Figure 3}

Genotypic and phenotypic characterization of virus isolated from mice following infection with T3SA- and T3SA+. (a) Viral genomic RNA was extracted from either homogenized livers of infected mice or second-passage lysate stocks. S1 gene segment cDNA's were amplified using RT-PCR (labeled at right as Primary and Secondary), and restriction enzyme digestion was performed using BstNI. Digested secondary PCR products (labeled at right as 355 bp and $190 \mathrm{bp}$ ) were visualized by agarose gel electrophoresis and ethidium bromide staining. Undigested $(U)$ and digested $(D)$ stock virions are shown along with infected livers from days 4,8 , and 12 after inoculation. The lanes labeled $\mathrm{M}$ were loaded with 100-bp markers. (b) Homogenized livers obtained 4, 8, and 12 days after inoculation were passaged in $L$ cells for 72 hours. $L$ cell lysate $(150 \mu \mathrm{l})$ was adsorbed to either $L$ cells or MEL cells $\left(2 \times 10^{5}\right)$ and incubated at $37^{\circ} \mathrm{C}$ for 24 hours. Viral titers at 0 hours and 24 hours were determined from liver samples obtained from three to four mice by plaque assay using $\mathrm{L}$ cells. Viral yields were calculated by dividing titer at 24 hours by titer at 0 hours, and average viral yields for all at 3 days after inoculation are shown. Error bars indicate SEM.

Virulence of T3SA- and T3SA+ following intracranial and peroral inoculation. To assess the neurovirulence of strains T3SA- and T3SA+, litters of newborn mice were inoculated intracranially with increasing doses (from 0.5 to $92 \mathrm{PFU}$ per mouse) of T3SA- or T3SA+, and mortality was monitored daily for 21 days after inoculation (Table 1). At the lowest dose tested, no animals died during the observation period, while all mice died after inoculation with either strain at the highest dose tested. At all intermediate doses, there was a trend toward increased lethality in litters infected with T3SA+. When these results were analyzed to calculate $\mathrm{LD}_{50}$ for each strain (52), the $\mathrm{LD}_{50}$ of T3SA+ was determined to be fourfold lower than that of T3SA- (2.5 PFU vs. 10 PFU). These results indicate that the capacity to bind sialic acid modestly increases reovirus virulence when virus is inoculated directly into the brain.

\section{Table 1}

Virulence of reovirus strains T3SA- and T3SA+ following intracranial inoculation

\section{Dose (PFU/mouse)} Percent lethality

$\begin{array}{lcc} & \text { T3SA- } & \text { T3SA+ } \\ 0.5 & 0 & 0 \\ 0.9 & 0 & 10 \\ 1.2 & 25 & 20 \\ 4 & 18 & 75 \\ 6 & 22 & 90 \\ 10 & 56 & 100 \\ 25 & 82 & 100 \\ 56 & 89 & 100 \\ 92 & 100 & 100 \\ \text { Calculated } \text { LD }_{50} & 10 \mathrm{PFU} & 2.5 \mathrm{PFU}\end{array}$

ND4 Swiss Webster mice, 2-3 days old, were inoculated intracranially with the indicated doses of T3SA- or T3SA+. Each row represents one litter of mice (eight to eleven animals). Litters were monitored for mortality for a period of 21 days after inoculation, at which point survivors appeared healthy. $\mathrm{LD}_{50}$ values were calculated according to the method of Reed and Muench (52). 
Table 2

Virulence of reovirus strains T3SA- and T3SA+ following peroral inoculation

\begin{tabular}{lcc}
\hline Dose (PFU/mouse) & \multicolumn{2}{c}{ Percent lethality } \\
& T3SA- & T3SA+ \\
$10^{1}$ & 0 & 0 \\
$10^{2}$ & 0 & 0 \\
$10^{3}$ & 30 & 42 \\
$10^{4}$ & 21 & 61 \\
$10^{5}$ & 80 & 63 \\
$10^{6}$ & 100 & 90 \\
$10^{7}$ & 100 & 100 \\
$10^{8}$ & 100 & 100 \\
Calculated LD $_{50}$ & $10^{4} \mathrm{PFU}$ & $10^{4} \mathrm{PFU}$ \\
\cline { 2 - 3 }
\end{tabular}

ND4 Swiss Webster mice, 2-3 days old, were inoculated perorally with the indicated doses of T3SA- or T3SA+. Each row represents one litter of mice (eight to eleven animals). Litters were monitored for mortality for a period of 21 days after inoculation, at which point survivors appeared healthy. $L_{50}$ values were calculated according to the method of Reed and Muench (52).

To determine the role of sialic acid binding in virulence following a natural route of infection, newborn mice were inoculated perorally with increasing doses $\left(10^{1}\right.$ to $10^{8} \mathrm{PFU}$ per mouse) of T3SA- or T3SA+ and monitored daily for mortality (Table 2). In contrast to results obtained following intracranial inoculation, we observed no difference in mortality between these strains following peroral inoculation. Together, these results indicate that the capacity to bind sialic acid does not make a major contribution to the virulence of T3 reovirus following peroral inoculation but may lead to enhanced injury to neural tissues following entry into the CNS.

Mice infected with T3SA+ develop OHE. During the course of these experiments, we noted that the majority of mice inoculated perorally with more than $10^{2} \mathrm{PFU}$ of T3SA+ developed OHE (Figure 4, $n=22$ of 29 litters displaying OHE). OHE was previously reported in association with reovirus infection of intrahepatic bile duct epithelium, resulting in biliary tract obstruction and fat malabsorption $(7,9-11)$. OHE developed in mice infected with T3SA+ between 10 and 14 days after inoculation. Closer inspection revealed that mice with OHE excreted fatty, acholic stools, with oily hair likely deriving from contact with lipid-rich stools in the bedding. Mice also appeared growth-retarded in comparison with those infected with T3SA-. OHE and acholic stools were never observed in litters infected with T3SA-, even at doses up to $10^{8} \mathrm{PFU}$ per mouse $(n=26$ litters receiving doses of greater than $10^{2} \mathrm{PFU}$ perorally). However, mice infected with either virus strain developed neurologic symptoms, and mortality associated with either infection appeared to be due to encephalitic disease. Mice surviving the initial infection ultimately cleared signs of OHE and recovered completely. Thus, mice infected with T3SA+ appear to develop signs of obstructive liver disease in addition to the encephalitis characteristic of $\mathrm{T} 3$ reovirus infection.
Serum alkaline phosphatase and bilirubin levels in mice following infection with T3SA- and T3SA+. To determine whether mice showed biochemical evidence of biliary obstruction, we assessed alkaline phosphatase and bilirubin levels in mice that were either mock infected or infected with T3SA- or T3SA+ (Figure 5). Neither biomarker of biliary tract injury was consistently elevated in mock-infected mice or mice infected with T3SA-. In contrast, levels of alkaline phosphatase were moderately increased in animals infected with T3SA+ on days 4 to 12 after inoculation. Importantly, serum bilirubin levels rose sharply in animals infected with T3SA+ on day 10 after inoculation, coincident with the development of oily hair and steatorrhea. These results indicate that OHE induced by $\mathrm{T} 3 \mathrm{SA}+$ is associated with biochemical signs of biliary tract injury.

Liver histopathology in mice following infection with T3SAand T3SA+. We next examined liver tissue of mice infected with T3SA- and T3SA+ for evidence of biliary tract inflammation (Figure 6). Eight days after peroral inoculation, liver from mice inoculated with T3SAcontained moderate periportal inflammatory infiltrates. However, like the bile ducts of mice inoculated with PBS (Figure 6a), bile ducts of mice infected with T3SA- were patent and showed no evidence of inflammation (Figure 6b). In contrast, livers from mice infected with T3SA+ contained marked portal lymphoid infiltrates, with bridging of adjacent portal triads and frequent encroachment on the central vein. Inflammatory cells appeared to cluster around the bile ducts, with evidence of bile duct necrosis observed in some sections (Figure 6, $c$ and d).

Immunohistochemical localization of reovirus antigen in bile duct epithelial cells. To directly test whether sialic acid binding confers tropism for bile duct epithelial cells, liver sections from mice infected with T3SA- and T3SA+ were stained for reovirus antigen (Figure 7). The timepoint of 6 days after inoculation was chosen for this analysis, since we reasoned that viral infection would precede the inflammation observed at 8 days after inoculation. Liver tissue of mice infected with T3SA- displayed occasional antigen-positive hepatocytes within the parenchyma, but bile duct epithelial

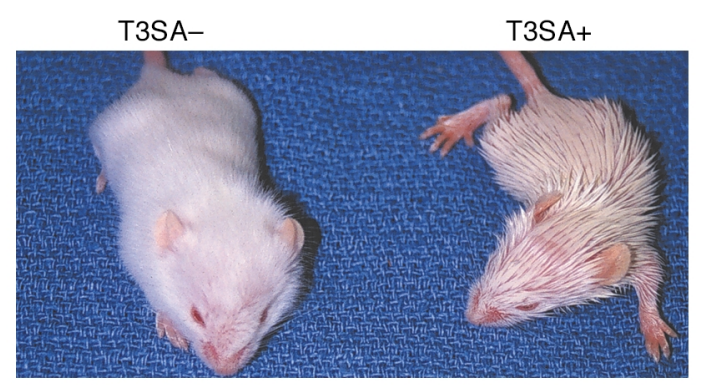

\section{Figure 4}

Mice infected with T3SA+ develop OHE. Two-day-old ND4 Swiss Webster mice were inoculated perorally with $1 \times 10^{4} \mathrm{PFU}$ of either T3SA- or T3SA+ and photographed 11 days after inoculation. 

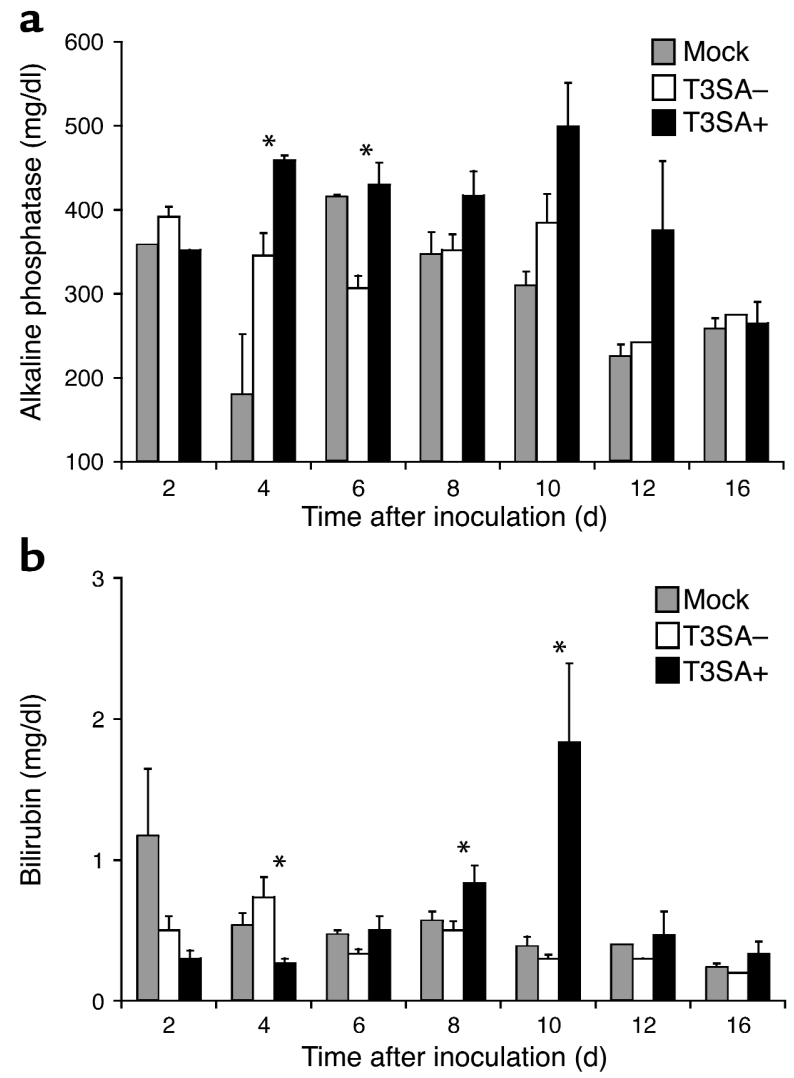

cells were consistently antigen-negative (Figure 7, a and $b$ ). In contrast, nearly every bile duct found in the liver of mice infected with T3SA+ displayed strong reovirus antigen staining in the cytoplasm of cuboidal bile duct epithelial cells (Figure 7, c and d). In most cases, infected bile duct tissue was also the focus of an inflammatory exudate. Differences in the patterns of inflammation induced by T3SA- and T3SA+ persisted at later timepoints, even when viral titers in liver tissue were equivalent (data not shown). These findings provide strong evidence that the capacity to bind sialic acid influences tropism of T3 reovirus to bile duct epithelium, which results in biliary tract inflammation and obstructive jaundice.

Binding of T3SA- and T3SA+ to a human biliary epithelial cell line. To test the hypothesis that tropism of T3SA+ for biliary epithelium observed in vivo is linked to the capacity of this strain to bind sialic acid on the biliary epithelial surface, we assessed the binding of T3SAand T3SA+ to Mz-Cha-1 cells (Figure 8). Mz-Cha-1 cells are immortalized human cholangiocarcinoma cells that retain many biochemical and ultrastructural characteristics of biliary epithelial cells (46). L cells, which do not require sialic acid binding for efficient infection (45), were used as a positive control for binding. $\mathrm{L}$ cells and $\mathrm{Mz}$-Cha- 1 cells were either mocktreated or treated with neuraminidase to remove cellsurface sialic acid and then incubated with increasing concentrations of T3SA- and T3SA+. The extent of virus binding was measured using polyclonal

\section{Figure 5}

Serum alkaline phosphatase and bilirubin levels in mice after infection with T3SA- and T3SA+. ND4 Swiss Webster mice, 2-3 days old, were inoculated perorally with $2.5 \times 10^{3} \mathrm{PFU}$ of either T3SA- or $\mathrm{T} 3 \mathrm{SA}+$. Mock-infected animals were inoculated perorally with PBS. At the indicated times after inoculation, mice were euthanized, blood was collected, and serum was separated by centrifugation. Total alkaline phosphatase (a) and bilirubin (b) levels were determined for each serum sample. Each bar represents an average enzyme level for one to four mice. Error bars indicate SEM. ${ }^{*} P<0.05$, T3SA-vs. T3SA+ by Student $t$ test.

anti-reovirus serum and flow cytometry. As anticipated, the binding of T3SA- and T3SA+ to L cells was equivalent, with binding of either strain easily detectable with as little as $10^{3}$ viral particles per cell. $\mathrm{T} 3 \mathrm{SA}+$ bound with slightly higher efficiency than T3SA- to L cells (three- to fivefold enhancement), and this increase in binding efficiency was eliminated by treatment with neuraminidase (Figure 8, a-d). However, the binding of T3SA- to Mz-Cha- 1 cells was minimal, even at the highest dose of virus tested $\left(10^{5}\right.$ particles per cell) (Figure 8e). In striking contrast, high-level, dose-dependent binding of T3SA+ to $\mathrm{Mz}-$ Cha- 1 cells was observed at all virus doses tested, and the level of binding of T3SA+ to Mz-Cha- 1 cells was 10- to 100-fold more efficient than that of T3SA- (Figure 8f). The efficiency of T3SA+ binding to Mz-Cha-1 cells was reduced to that of T3SA- by neuraminidase treatment, indicating that the observed binding is specific and dependent on sialic acid (Figure 8, $g$ and $h$ ). These findings indicate that the capacity to bind sialic acid results in a dramatic enhancement of reovirus attachment to biliary epithelial cells and strongly suggest that the capacity to bind this carbohydrate is required for infection of this cell type in vivo.
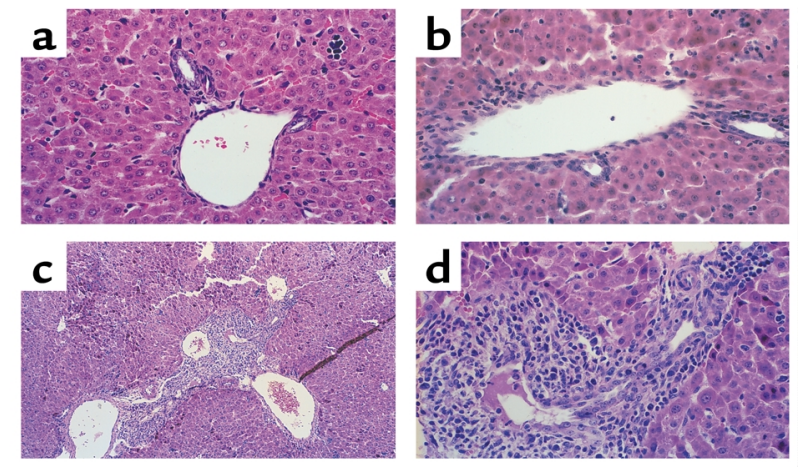

Figure 6

Liver histopathology in mice following infection with T3SA- and T3SA+. ND4 Swiss Webster mice, 2-3 days old, were inoculated perorally with PBS (a) or $2.5 \times 10^{3}$ PFU of either T3SA- (b) or $\mathrm{T} 3 \mathrm{SA}+(\mathbf{c}$ and $\mathbf{d})$. At 8 days after inoculation, liver tissue was harvested, embedded in paraffin, thin-sectioned, and stained with hematoxylin and eosin. Sections from approximately ten mice infected with each virus were examined and showed qualitatively similar results. Photomicrographs were prepared at final magnifications of $\times 100(\mathbf{c})$ or $\times 400(\mathbf{a}, \mathbf{b}$, and $\mathbf{d})$. 

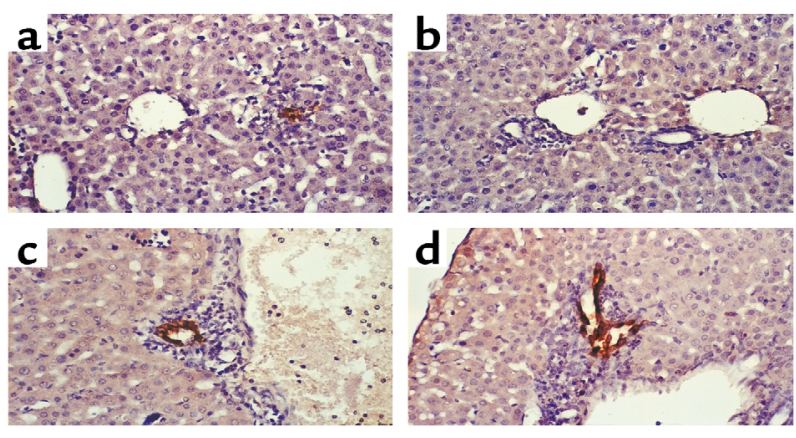

\section{Figure 7}

Immunohistochemical localization of reovirus antigen in bile duct epithelial cells. ND4 Swiss Webster mice, 2-3 days old, were inoculated perorally with $2.5 \times 10^{3} \mathrm{PFU}$ of either T3SA- $(\mathbf{a}$ and $\mathbf{b})$ or T3SA+ (c and d). Six days after inoculation, liver tissue was harvested, embedded in paraffin, thin-sectioned, and stained for reovirus antigen using rabbit anti-reovirus serum and horseradish peroxidase. Dark brown staining indicates reovirus antigen. Sections from approximately ten mice infected with each virus were examined and showed qualitatively similar results. Photomicrographs were prepared at $\times 400$ final magnification. Representative sections from two separate animals are shown.

\section{Discussion}

Results of experiments described here represent the first characterization of the role of carbohydrate binding in reovirus tropism and pathogenesis in the infected host. We report that sialic acid binding enhances spread of $\mathrm{T} 3$ reovirus from the intestine to distal organs, including the spleen, liver, and brain. In addition, we demonstrate that the capacity to bind sialic acid is required for reovirus tropism for bile duct epithelial cells and ensuing biliary tract destruction. These findings support the hypothesis that binding to carbohydrate modulates the systemic nature of reovirus disease by altering viral tropism at the level of virus attachment. In addition, these findings indicate that ubiquitous cell-surface carbohydrates, such as sialic acid, are capable of determining specificity of reovirus tropism not only in cultured cells but also in the infected animal.

The capacity to bind sialic acid enhances reovirus dissemination from the intestine to distal organs. Comparison of viral titers in the intestine, spleen, liver, and brain

\footnotetext{
Figure 8

Binding of T3SA- and T3SA+ to $L$ cells and Mz-Cha- 1 cells. $L$ cells (a-d) or Mz-Cha-1 biliary epithelial cells (e-h) were either mocktreated ( $\mathbf{a}, \mathbf{b}, \mathbf{e}$, and $\mathbf{f})$ or treated with $\mathbf{C}$. perfringens neuraminidase to remove cell-surface terminal sialic acid residues ( $\mathbf{c}, \mathbf{d}, \mathbf{g}$, and $\mathbf{h})$. Cells were incubated with 0 (black line), $10^{3}$ (red line), $10^{4}$ (green line), or $10^{5}$ (blue line) particles per cell of T3SA- $(\mathbf{a}, \mathbf{c}, \mathbf{e}$, and $\mathbf{g}$ ) or T3SA+ $(\mathbf{b}, \mathbf{d}, \mathbf{f}$, and $\mathbf{h})$. Unbound virus was removed, and bound virus on the cell surface was detected using rabbit anti-reovirus serum followed by phycoerythrin-labeled goat anti-rabbit Fab's. Histograms represent data gated on total live-cell populations. Shown are representative histograms from one experiment ( $L$ cells) or two experiments (Mz-Cha-1 cells). Preimmune rabbit serum caused less than a threefold increase in fluorescence in the presence of $10^{6}$ particles per cell of virus (data not shown).
}

after peroral or intracranial inoculation indicates that the capacity to bind sialic acid can enhance reovirus spread from the primary site of replication to distal organs. One mechanism for enhanced spread might involve sialic acid-mediated tropism of T3SA+ for a circulating cell type. It is known that sialic acid-binding T3 strains replicate within submucosal lymphoid populations in the intestine $(4,53,54)$, and it is possible that $\mathrm{T} 3 \mathrm{SA}+$ preferentially infects circulating monocytes, lymphocytes, or dendritic cells. The observation that titers of T3SA+ increase early (day 4 after inoculation) in the spleen, a highly vascular organ, suggests that this strain may be more efficient at entering the host blood stream following intestinal replication. This early boost in hematogenous titer may result in rapid viral seeding of distal organs, including liver and brain. However, higher titers of $\mathrm{T} 3 \mathrm{SA}+$ detected in liver and brain cannot be solely due to an increase in hematogenous titer, since T3SA+ produces significantly higher titers in these organs at day 8 after inoculation, at which time titers of T3SAand $\mathrm{T} 3 \mathrm{SA}+$ in the spleen are equivalent. In the case of enhanced spread of T3SA+ to the CNS, it is possible that this strain manifests an increased efficiency to infect vagus neurons, thereby accelerating its entry into the brain stem (4).

Surprisingly, although T3SA+ demonstrates an enhanced capacity to spread to the CNS, neither its virulence nor kinetics of lethality following peroral inoculation is significantly altered in comparison to T3SA-. Because of its delayed dissemination from the intestine, we tested whether T3SA- mediates spread by mutating to a virus capable of binding sialic acid. Amplification of S1 gene segment cDNA's by RT-PCR and digestion with BstNI reveals that T3SA- does not
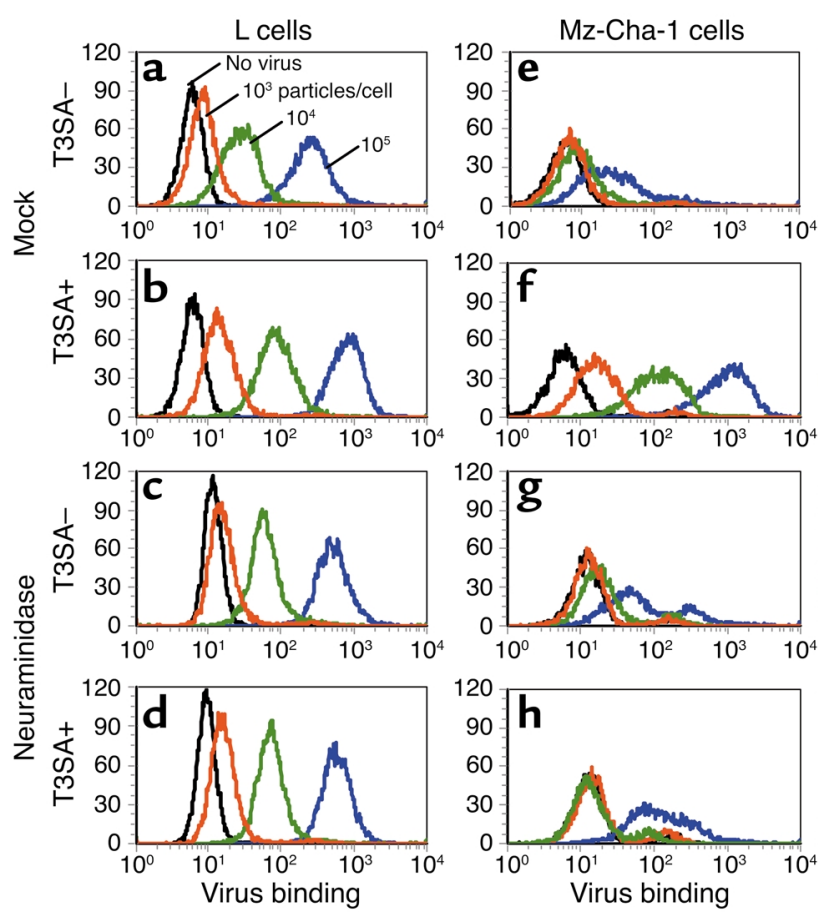
develop the point mutation that confers sialic acid binding. Furthermore, viruses isolated from infected mice retain their respective parental sialic acid-binding phenotypes, indicating that these strains are genetically and phenotypically stable in vivo. The lack of difference in mortality exhibited by T3SA- and T3SA+ may reflect the fact that mortality studies lack the sensitivity to detect differences in the type of disease that result in death of the host. This possibility seems particularly likely in the case of T3SA- and T3SA+ virulence since the pathogenesis of T3SA- seems limited to viral encephalitis, whereas T3SA+ is also capable of causing obstructive liver disease. Thus, although T3SA- and T3SA+ produce mortality with equivalent efficiency, they cause dramatically different clinical syndromes, as reflected in their differential tropism for bile duct epithelium.

The capacity to bind sialic acid is required for reovirus replication in bile duct epithelium. Use of sialic acid as a coreceptor clearly affects reovirus tropism for specific cells in the host, as evidenced by the striking tropism of T3SA+ for bile duct epithelial cells observed in this study. The observation that T3SA+ but not T3SA- is capable of efficient binding to human biliary epithelial cells suggests that reovirus biliary tropism is mediated directly at the biliary epithelial cell surface. However, the precise mechanism by which sialic acid binding targets reovirus to bile duct epithelium remains to be determined. Of note, bile duct epithelial cells express high levels of JAM1 (C. Parkos, personal communication), the proteinaceous receptor used by both T3SA- and T3SA+ (41). In addition, our preliminary studies indicate that highaffinity binding of T3SA + to human biliary epithelial Mz-Cha- 1 cells requires virus binding to both sialic acid and JAM1 (E.S. Barton and T.S. Dermody, unpublished observation). The preferential infection of bile duct epithelium by T3SA+ therefore suggests that sialic acid binding enhances virus infection through adhesion to the cell surface, where JAM1 is accessed. Alternatively, since the bile duct epithelium is isolated from direct contact with the circulation by the bile canalicular system (55), it is possible that T3SA+ bile duct tropism is determined by the capacity to infect subsets of hepatocytes that line these canals. Infection of such cells would allow excretion of reovirus particles into bile canaliculi and subsequent bile duct infection. It is also possible that T3SA+ gains access to intrahepatic bile ducts through retrograde infection from the duodenum along the common bile duct. Thus, our data demonstrate an important role for sialic acid binding in bile duct tropism but do not define whether the critical sialic acid residues bound by reovirus reside on the biliary epithelial surface or on other host cells.

In a previous study of reovirus-induced biliary disease, the $\sigma 1$ protein was implicated as a determinant of bile duct tropism (7). Reovirus strains T3C31 and T3C87 were capable of inducing OHE, and interestingly, both of these strains bind sialic acid (39). Two additional strains, T3C43 and T3C84, did not induce OHE, and neither binds this carbohydrate (39). However, the correlation of sialic acid binding and OHE was not absolute because sialic acid-binding strains T3D and T3C9 did not induce OHE. These results suggest that sialic acid binding capacity is necessary but not sufficient for bile duct tropism, a conclusion that seems especially likely in the case of T3D. Although T3D is capable of binding sialic acid (39), it is essentially noninfectious following peroral inoculation (56), which makes it difficult to draw conclusions about its pathogenic phenotypes following inoculation by this route. In addition to the established role of the $S 1$ gene in reovirus-induced biliary tract infection, a secondary effect of the viral M2 gene, which encodes an outer-capsid protein involved in viral entry, has been reported (8). Taken together, these results point to an important role for early steps in the reovirus life cycle, specifically attachment and entry, in controlling viral tropism for bile duct epithelium.

Host factors are also capable of modulating the capacity of reovirus to trigger biliary tract disease. Reovirus strain T3C9, which does not cause OHE in normal newborn mice, is capable of doing so if mice are first depleted of $\mathrm{CD}^{+}$and $\mathrm{CD}^{+} \mathrm{T}$ lymphocytes (57), indicating that the host immune response can inhibit biliary tract infection with a strain that is capable of binding sialic acid. This observation provides additional evidence that infection with sialic acid-binding reovirus is not sufficient to produce biliary tract disease. Instead, bile duct injury appears to be a complex interaction between tropism of the infecting strain, efficiency of the antiviral immune response, and perhaps other additional viral and host factors.

Reovirus and biliary atresia. Although the etiology of biliary atresia is not known, the sudden onset and progressive inflammation of this disease strongly suggest a role for an infectious agent. Biliary atresia affects infants in the first few months of life, whereas the average age of first reovirus infection is thought to be after the first year of life (58). These observations suggest that only rarely would an infant experience initial reovirus infection during the period of risk for biliary atresia, perhaps due to protection from maternal anti-reovirus antibody, in accordance with the low overall incidence $(1: 10,000$ to 1:15,000) of biliary atresia.

Numerous serologic studies have attempted to identify viral inducers of biliary atresia. Some studies have demonstrated a correlation between the presence of anti-reovirus antibodies and biliary atresia, while others have not (16-23). Our findings may explain the discordance between anti-reovirus seropositivity and biliary atresia. If sialic acid-binding $\mathrm{T} 3$ strains preferentially infect bile duct tissue, then infants acquiring infection with T1, T2, or non-sialic acid-binding T3 strains would be at less risk for biliary atresia. In addition, due to the antigenic similarity of other reovirus 
proteins across serotypes (1), these individuals would likely be protected from future infection with reovirus strains capable of infecting bile duct epithelial cells. Furthermore, breastfed infants whose mothers transferred sufficient levels of anti-reovirus antibodies (raised against any strain) might also be protected from such an infection.

In the most detailed study to date that implicates reovirus infection as a potential cause of bile duct injury in humans, liver and bile duct tissue samples from patients with biliary atresia or a related biliary tract disease, choledochal cysts, were examined for reovirus RNA by RT-PCR amplification of the reovirus $L 1$ gene (24). As controls, samples of liver tissue were taken from patients with non-biliary tract liver disease. A highly statistically significant correlation was found between the presence of reovirus $L 1$ RNA in tissue samples and obstructive liver disease. These results indicate that reovirus infection is strongly associated with biliary atresia and choledochal cysts in humans, suggesting that reovirus infection of the biliary tract may play a causal role in these processes. Since the $S 1$ gene was not amplified from the tissue samples used in this study, it was not possible to determine whether viral sialic acid binding capacity is correlated with human biliary tract disease. Such a study would be informative and permit a direct test of the genetic linkage of sialic acid binding by reovirus and biliary atresia.

Results presented here firmly establish a role for sialic acid binding in reovirus tropism and pathogenesis in the host and suggest that differences in the kinetics of reovirus attachment to host cells have important consequences in the infected animal. Further comparison of T3SA- and T3SA+ neural and hematogenous spread, CNS tropism, and bile duct tropism should enable a molecular dissection of the multistep attachment processes that regulate reovirus-induced disease.

\section{Acknowledgments}

We thank Kelly Parman of the Vanderbilt University School of Medicine (VUSM) Mouse Pathology Core Lab for tissue sectioning and Greg Hanley and Joan Richerson for expert veterinary care. We thank Greg Fitz at the University of Colorado for graciously providing us with the Mz-Cha-1 cells. We are grateful to Jim Chappell and Tim Peters for review of the manuscript and to Brent Weedman for preparation of the histology figures. This research was supported by a grant from the National Science Foundation (to E.S. Barton); NIH Public Health Service awards T32 AI07474 (to B.E. Youree and T. Valyi-Nagy), T32 GM07347 (to D.H. Ebert), T32 CA-09385 (to J.C. Forrest), and R01 AI-38296; the Vanderbilt University Research Council (to E.S. Barton and J.C. Forrest); and the Elizabeth B. Lamb Center for Pediatric Research. Additional support was provided by NIH Public Health Service awards CA-68485 for the Vanderbilt-Ingram Cancer Center and DK-20593 for the Diabetes Research and Training Center of VUSM.
1. Tyler, K.L. 2001. Mammalian reoviruses. In Fields virology. 4th edition. D.M. Knipe and P.M. Howley, editors. Lippincott-Raven Publishers. Philadelphia, Pennsylvania, USA. 1729-1945.

2. Virgin, H.W., Tyler, K.L., and Dermody, T.S. 1997. Reovirus. In Viral pathogenesis. N. Nathanson, editor. Lippincott-Raven Publishers. Philadelphia, Pennsylvania, USA. 669-699.

3. Tardieu, M., and Weiner, H.L. 1982. Viral receptors on isolated murine and human ependymal cells. Science. 215:419-421.

4. Morrison, L.A., Sidman, R.L., and Fields, B.N. 1991. Direct spread of reovirus from the intestinal lumen to the central nervous system through vagal autonomic nerve fibers. Proc. Natl. Acad. Sci. U. S. A. 88:3852-3856.

5. Kaye, K.M., Spriggs, D.R., Bassel-Duby, R., Fields, B.N., and Tyler, K.L. 1986. Genetic basis for altered pathogenesis of an immune-selected antigenic variant of reovirus type 3 (Dearing). J. Virol. 59:90-97.

6. Oberhaus, S.M., Smith, R.L., Clayton, G.H., Dermody, T.S., and Tyler, K.L. 1997. Reovirus infection and tissue injury in the mouse central nervous system are associated with apoptosis. J. Virol. 71:2100-2106.

7. Wilson, G.A.R., Morrison, L.A., and Fields, B.N. 1994. Association of the reovirus $\mathrm{S} 1$ gene with serotype 3 -induced biliary atresia in mice. J. Virol. 68:6458-6465.

8. Derrien, M., Hooper, J.W., and Fields, B.N. 2003. The M2 gene segment is involved in the capacity of reovirus type 3 Abney to induce the oily fur syndrome in neonatal mice, a S1 gene segment-associated phenotype. Virology. 305:25-30.

9. Papadimitriou, J.M. 1968. The biliary tract in acute murine reovirus 3 infection. Am. J. Pathol. 52:595-601.

10. Phillips, P.A., Keast, D., Papadimitriou, J.M., Walters, M.N., and Stanley, N.F. 1969. Chronic obstructive jaundice induced by reovirus type 3 in weanling mice. Pathology. 1:193-203.

11. Parashar, K., Tarlow, M.J., and McCrae, M.A. 1992. Experimental reovirus type 3-induced murine biliary tract disease. J. Pediatr. Surg. 27:843-847.

12. Middlesworth, W., and Altman, R.P. 1997. Biliary atresia. Curr. Opin. Pediatr. 9:265-269.

13. Bates, M.D., Bucuvalas, J.C., Alonso, M.H., and Ryckman, F.C. 1998. Biliary atresia: pathogenesis and treatment. Semin. Liver Dis. 18:281-293.

14. Lefkowitch, J.H. 1998. Biliary atresia. Mayo Clin. Proc. 73:90-95.

15. Narkewicz, M.R. 2001. Biliary atresia: an update on our understanding of the disorder. Curr. Opin. Pediatr. 13:435-440.

16. Brown, W.R., et al. 1988. Lack of correlation between infection with reovirus 3 and extrahepatic biliary. J. Pediatr. 113:670-676.

17. Brown, W.R. 1990. Lack of confirmation of the association of reovirus 3 and biliary atresia: methodological differences. Hepatology. 12:1254-1255.

18. Dussaix, E., Hadchouel, M., Tardieu, M., and Alagille, D. 1984. Biliary atresia and reovirus type 3 infection. N. Engl. J. Med. 310:658.

19. Bangaru, B., Morecki, R., Glaser, J.H., Gartner, L.M., and Horwitz, M.S. 1980. Comparative studies of biliary atresia in human newborn and reovirus-induced cholangitis in weanling mice. Lab. Invest. 43:456-462.

20. Minuk, G.Y., et al. 1987. Reovirus type 3 infection in patients with primary biliary cirrhosis and primary sclerosing cholangitis. J. Hepatol. 5:8-13.

21. Morecki, R., Glaser, J.H., Cho, S., Balistreri, W.F., and Horwitz, M.S. 1982. Biliary atresia and reovirus type 3 infection. N. Engl. J. Med. 307:481-484.

22. Morecki, R., Glaser, J.H., Johnson, A.B., and Kress, Y. 1984. Detection of reovirus type 3 in porta hepatis of an infant with extrahepatic biliary atresia. Ultrastructural and immunocytochemical study. Hepatology. 4:1137-1142.

23. Morecki, R., and Glaser, J. 1989. Reovirus 3 and neonatal biliary disease: discussion of divergent results. Hepatology. 10:515-517.

24. Tyler, K.L., et al. 1998. Detection of reovirus RNA in hepatobiliary tissues from patients with extrahepatic biliary atresia and choledochal cysts. Hepatology. 27:1475-1482.

25. Kauffman, R.S., Wolf, J.L., Finberg, R., Trier, J.S., and Fields, B.N. 1983. The sigma 1 protein determines the extent of spread of reovirus from the gastrointestinal tract of mice. Virology. 124:403-410.

26. Tyler, K.L., McPhee, D.A., and Fields, B.N. 1986. Distinct pathways of viral spread in the host determined by reovirus S1 gene segment. Science. 233:770-774.

27. Weiner, H.L., Powers, M.L., and Fields, B.N. 1980. Absolute linkage of virulence and central nervous system tropism of reoviruses to viral hemagglutinin. J. Infect. Dis. 141:609-616.

28. Weiner, H.L., Drayna, D., Averill, D.R., Jr., and Fields, B.N. 1977. Molecular basis of reovirus virulence: role of the S1 gene. Proc. Natl. Acad. Sci. U. S. A. 74:5744-5748.

29. Weiner, H.L., Ault, K.A., and Fields, B.N. 1980. Interaction of reovirus with cell surface receptors. I. Murine and human lymphocytes have a receptor for the hemagglutinin of reovirus type 3. J. Immunol. 124:2143-2148.

30. Lee, P.W., Hayes, E.C., and Joklik, W.K. 1981. Protein $\sigma 1$ is the reovirus cell attachment protein. Virology. 108:156-163.

31. Burness, A.T.H. 1981. Glycophorin and sialylated components as receptors 
for viruses. In Virus receptors. K. Lonberg-Holm and L. Philipson, editors. Chapman and Hall. London, United Kingdom. 63-84.

32. Gentsch, J.R., and Pacitti, A.F. 1985. Effect of neuraminidase treatment of cells and effect of soluble glycoproteins on type 3 reovirus attachment to murine L cells. J. Virol. 56:356-364.

33. Gentsch,J.R., and Pacitti, A.F. 1987. Differential interaction of reovirus type 3 with sialylated receptor components on animal cells. Virology. 161:245-248.

34. Paul, R.W., and Lee, P.W.K. 1987. Glycophorin is the reovirus receptor on human erythrocytes. Virology. 159:94-101.

35. Paul, R.W., Choi, A.H., and Lee, P.W.K. 1989. The $\alpha$-anomeric form of sialic acid is the minimal receptor determinant recognized by reovirus. Virology. 172:382-385.

36. Rubin, D.H., et al. 1992. Receptor utilization by reovirus type 3: distinct binding sites on thymoma and fibroblast cell lines result in differential compartmentalization of virions. Microb. Pathog. 12:351-365.

37. Chappell, J.D., Gunn, V.L., Wetzel, J.D., Baer, G.S., and Dermody, T.S 1997. Mutations in type 3 reovirus that determine binding to sialic acid are contained in the fibrous tail domain of viral attachment protein $\sigma 1$. J. Virol. 71:1834-1841.

38. Chappell, J.D., Duong, J.L., Wright, B.W., and Dermody, T.S. 2000. Identification of carbohydrate-binding domains in the attachment proteins of type 1 and type 3 reoviruses. J. Virol. 74:8472-8479.

39. Dermody, T.S., Nibert, M.L., Bassel-Duby, R., and Fields, B.N. 1990. A o1 region important for hemagglutination by serotype 3 reovirus strains. J. Virol. 64:5173-5176.

40. Lerner, A.M., Cherry, J.D., and Finland, M. 1963. Haemagglutination with reoviruses. Virology. 19:58-65.

41. Barton, E.S., et al. 2001. Junction adhesion molecule is a receptor for reovirus. Cell. 104:441-451.

42. Liu, Y., et al. 2000. Human junction adhesion molecule regulates tight junction resealing in epithelia. J. Cell Sci. 113:1-11.

43. Martin-Padura, I., et al. 1998. Junctional adhesion molecule, a novel member of the immunoglobulin superfamily that distributes at intercellular junctions and modulates monocyte transmigration. J. Cell Biol. 142:117-127.

44. Ostermann, G., Weber, K.S., Zernecke, A., Schroder, A., and Weber, C. 2002. JAM-1 is a ligand of the beta(2) integrin LFA-1 involved in transendothelial migration of leukocytes. Nat. Immunol. 3:151-158.
45. Barton, E.S., Connolly, J.L., Forrest, J.C., Chappell, J.D., and Dermody, T.S. 2001. Utilization of sialic acid as a coreceptor enhances reovirus attachment by multistep adhesion strengthening. J. Biol. Chem. 276:2200-2211

46. Knuth, A., et al. 1985. Biliary adenocarcinoma. Characterisation of three new human tumor cell lines. J. Hepatol. 1:579-596.

47. Furlong, D.B., Nibert, M.L., and Fields, B.N. 1988. Sigma 1 protein of mammalian reoviruses extends from the surfaces of viral particles. J. Virol. 62:246-256.

48. Connolly, J.L., Barton, E.S., and Dermody, T.S. 2001. Reovirus binding to cell surface sialic acid potentiates virus-induced apoptosis. J. Virol. 75:4029-4039.

49. Rubin, D.H., and Fields, B.N. 1980. Molecular basis of reovirus virulence: role of the M2 gene. J. Exp. Med. 152:853-868.

50. Tyler, K.L., Bronson, R.T., Byers, K.B., and Fields, B.N. 1985. Molecular basis of viral neurotropism: experimental reovirus infection. Neurology. 35:88-92.

51. Virgin, H.W., IV, Bassel-Duby, R., Fields, B.N., and Tyler, K.L. 1988. Antibody protects against lethal infection with the neurally spreading reovirus type 3 (Dearing). J. Virol. 62:4594-4604.

52. Reed, L.J., and Muench, H. 1938. A simple method of estimating fifty per cent endpoints. Am. J. Hyg. 27:493-497.

53. Amerongen, H.M., Wilson, G.A.R., Fields, B.N., and Neutra, M.R. 1994. Proteolytic processing of reovirus is required for adherence to intestinal M cells. J. Virol. 68:8428-8432.

54. Wolf, J.L., et al. 1981. Intestinal M cells: a pathway of entry of reovirus into the host. Science. 212:471-472.

55. Oldham-Ott, C.K., and Gilloteaux, J. 1997. Comparative morphology of the gallbladder and biliary tract in vertebrates: variation in structure, homology in function and gallstones. Microsc. Res. Tech. 38:571-597.

56. Bodkin, D.K., and Fields, B.N. 1989. Growth and survival of reovirus in intestinal tissue: role of the L2 and S1 genes. J. Virol. 63:1188-1193.

57. Virgin, H.W., and Tyler, K.L. 1991. Role of immune cells in protection against and control of reovirus infection in neonatal mice. J. Virol. 65:5157-5164

58. Lerner, A.M., Cherry, J.D., Klein, J.O., and Finland, M. 1962. Infections with reoviruses. N. Engl. J. Med. 267:947-952. 